\title{
HTL meets Industrie 4.0
}

\section{J. Wagner}

Online publiziert am 9. Jänner 2018

C) Springer-Verlag GmbH Austria, ein Teil von Springer Nature 2018

Direktoren und Abteilungsvorstände der HTLs aus ganz Österreich tagten mit Vertretern des Gewerbes und der Industrie unter der Leitung des Bundesministeriums für Bildung (BMB) und der Pädagogische Hochschule NÖ (PH-NÖ) zwei Tage in der HTL St. Pölten (Abb. 1).

Das Thema Industrie 4.0 als ein zentraler Fokus in der Innovation der Wirtschaft wurde in den Mittelpunkt der Tagung von 180 Führungskräften aus dem Bereich der HTL gestellt. Sektionschef Dr. Christian Dorninger lud am 7. und 8. März 2017 in die HTL St. Pölten zu dieser Fachkonferenz, die von LSI HR Dr. Wilhelm König moderiert wurde, ein. In seiner Begrüßung bezeichnete der amtsführende Präsident des LSR, Mag. Johann Heuras, die Berufsbildenden Schulen als einen überaus wichtigen und erfolgreichen Schultyp in Österreich und betonte deren großes Ansehen im Ausland. SC Dr. Dorninger sprach in seinem Statement den aktuellen Stand der Bildungsreform an, danach ging MR Dipl.-Ing. Wolfgang Scharl vom BMB auf die Notwendigkeit der Auseinandersetzung mit dem Thema Industrie 4.0 in der HTL 4.0 ein (Abb. 2).

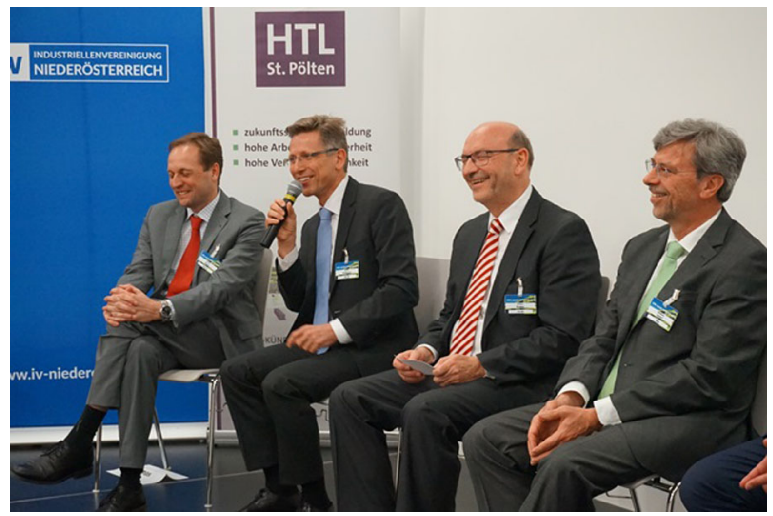

Abb. 1. HTL-Führungskräfte-Tagung mit Vertretern aus Gewerbe, Industrie, BMB und PH-NÖ

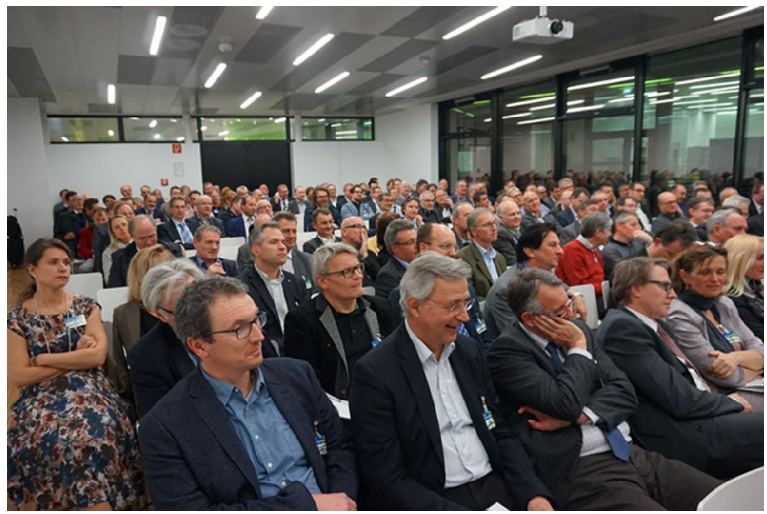

Abb. 2. Das Thema Industrie 4.0 stand im Fokus der Tagung von 180 Führungskräften aus dem Bereich der HTL
Anschließend standen in verschiedenen Vorträgen die Anforderungen und Erwartungshaltungen der Firmen im Vordergrund, wobei ein generell positives Bild der HTL-Absolventen in der Wirtschaft gezeichnet wurde. KR Veith Schmid-Schmidsfelden von der WKO Industrie ging ebenso wie die anderen Vertreter der Industrie auf den Begriff "lebenslanges Lernen" ein. In den einzelnen Beiträgen versuchten die Referenten, ihr Bild von Industrie 4.0 darzulegen, wobei die Vernetzung das zentrale Thema der verschiedenen technischen Fachrichtungen war. Daraus entwickelte sich in der anschließenden Podiumsdiskussion mit Vertretern von Magna power train, Audi/TH Ingolstadt, FH St. Pölten und Siemens AG, unter der Moderation von VR Dr. Norbert Kraker, ein reger Meinungsaustausch.

Im Abendprogramm legte Univ.-Prof. Dr. Rudolf Taschner auf bekannt launige Art seine Sichtweise von Industrie 4.0 im Zusammenhang mit der Mathematik dar. Somit war die Basis für die Workshops am zweiten Tag der Konferenz geschaffen. Als Eröffnung gab VR Kraker einen Überblick über die Struktur der Aus-, Fort- und Weiterbildung an der PH-NÖ für die Kollegenschaft der HTL und einen Überblick über die neu geschaffenen Arbeitseinheiten. MR Mag. Wolfgang Pachatz vom BMB versuchte, die Ziele der HTL für die Industrie 4.0 zu definieren und ging insbesondere auf die internationalen Erfolge dieser einzigartigen Schulform ein. Anschließend moderierten die Leiter der Fachwissenschaftlichen Zentren der PHNÖ sechs parallele Workshops. Dabei wurden die Visionen für HTL 4.0 erörtert, die Machbarkeit diskutiert und Notwendigkeiten für die Umsetzung definiert. Im Plenum der 180 mitwirkenden Führungskräfte gab es eine abschließende Diskussion mit der Zusage des $\mathrm{BMB}$, den Schulen die volle Unterstützung zukommen zu lassen und weitere Veranstaltungen zu diesem Thema durchzuführen (Abb. 3).

Als Resümee dieser von der HTL St. Pölten gemeinsam mit der $\mathrm{PH}-\mathrm{NO}$ hervorragend organisierten Fachkonferenz kann festgestellt werden, dass die Vision HTL 4.0 schon in verschiedenen Bereichen Realität ist und andere Bereiche sich auf einem sehr innovativen Weg in diese Richtung bewegen.

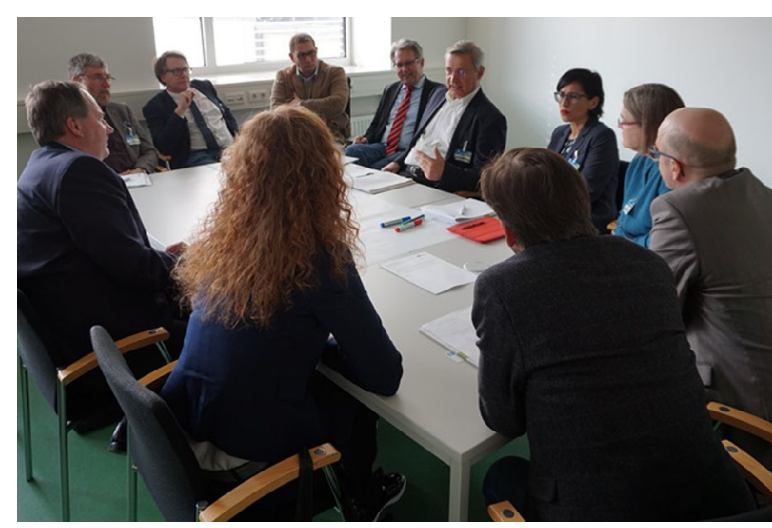

Abb. 3. Attraktive Workshops zum Thema "Visionen für HTL 4.0"

Wagner, Johann, Pädagogische Hochschule NÖ, Dechant-Pfeiffer-Straße 3, 2020 Hollabrunn, Österreich (E-Mail: johann.wagner@ph-noe.ac.at) 